\title{
Instrumentation of soil columns for time-lapse monitoring of the phenomenon of capillary rise through spontaneous potential, soil moisture sensor, electrical resistivity, and GPR measurements
}

\author{
Manuelle Santos Góis ${ }^{1 *}$, Moara Maria Oliveira de Matos $^{2}$, Nicolas Ohofugi Guimarães ${ }^{1}$, Katherin Rocio Cano Bezerra da \\ Costa $^{1}$, Gabriela Rodrigues Moreira ${ }^{1}$, Ana Carla Netto da Silva ${ }^{1}$, and André Luís Brasil Cavalcante ${ }^{1}$
}

${ }^{1}$ Department of Civil and Environmental Engineering, University of Brasilia, Zip Code 70910-900, Brazil

${ }^{2}$ Institute of Geosciences, University of Brasilia, Zip Code 70910-900, Brazil

\begin{abstract}
The continuous monitoring of capillary rise via indirect measures aims to predict and generate alerts regarding the soil mass deformations, transport leachate from landfills to the soil surface, and carry salts that can damage buildings. Through time-lapse monitoring of the electromagnetic wave's electrical potential and speed, it is possible to correlate via petrophysical relations the measures of electrical potential, electrical resistivity, and dielectric permittivity to the volumetric water content and capillary height. For this, four acrylic columns filled with civil construction material were instrumented. Column 1 - silver electrodes to measure the potential difference with a bench multimeter that measures the spontaneous potential generated by water flow. Column 2 - low-cost soil moisture sensors that measured the electrical potential and converted to bits. Column 3 - resistivimeter that measured the voltage and that was later converted to electrical resistivity and, Column $4-2.6 \mathrm{GHz}$ antenna that measured the speed of the electromagnetic wave that was later converted into dielectric permittivity. The instrumentation assembled proved to be satisfactory to monitor the phenomenon. The monitoring lasted $187 \mathrm{~h}$, and it was found that the maximum capillary height remained constant for a long time.
\end{abstract}

\section{Introduction}

A wide variety of technological applications, such as oil extraction through porous rocks, or ink-jet printing, is fundamentally dependent on capillary flows. The capillary rise phenomenon can be observed in many applied situations affecting the industry, infrastructure, agriculture, and environmental remediation.

In agriculture, this phenomenon helps in irrigating plants and is responsible for transporting water with nutrients.

Considering the geotechnical applications during the design of foundations, this phenomenon can alter some soils' mechanical properties by changes in pressure and hydraulic load. It can contribute to the consolidation of the soils that support the buildings and, when there are ionic exchanges, the capillary water can damage the concrete and steel of the foundations of the buildings. It can bring the landfill leach back to the surface.

In the construction of landfills and pavements, the water that rises through capillarity tends to compromise the pavements' durability; capillary siphoning can occur in dams: capillary water can pass through impermeable barriers and generate percolation through the dam. In this paper, the objective is to describe the phenomenon of capillary rise through indirect measures. With timelapse monitoring of the electrical potential and speed of the electromagnetic wave, it is possible to correlate via petrophysical relations the measures of electrical potential, electrical resistivity, and dielectric permittivity to the volumetric water content and capillary height (Revil et al. 2003 [1], Loeffler \& Bano 2004 [2], Jol 2009 [3], Franzoni \& Bandini 2012 [4], Klenk et al. 2015 [5], Revil et al. 2017 [6], Liu et al. 2017 [7], Oliveti \& Cardarelli 2019 [8], Gois et al. 2019 [9], Gois et al. 2020 [10]) however, few studies relate the indirect measures to capillary height for transient monitoring of long test periods due to the divergence of existing solutions for capillary height in these conditions.

\section{Capillary Rise at Porous Media}

The capillary front's evolution tends asymptotically towards an equilibrium height, for which the capillary and gravitational forces balance each other in static equilibrium. At the beginning of the capillary rise, the flow regime is linear, for which the gravity contribution can be neglected. In that situation, capillary forces are balanced only by viscous forces.

Resuming up along the z-direction, the forces driving a Hagen-Poiseuille flow in a porous media, the balance of capillary, gravity, viscous, and inertial forces gives (left to right) Eq.(1) Fries and Dreyer, 2008 [11].

$$
\frac{2 \mathrm{~T} \cos (\beta)}{\mathrm{r}}=\rho_{\mathrm{w}} \mathrm{gz}+\frac{8 \mu \mathrm{zz}}{\mathrm{r}^{2}}+\rho \frac{\mathrm{d}(\mathrm{zz})}{\mathrm{dt}}
$$

\footnotetext{
${ }^{*}$ Corresponding author: manuellegeo@gmail.com
} 
where $\dot{z}=d z / d t, z(t)$ is the height of capillary rise [L] at time $t[T], r$ is the equivalent radius of the capillary $[\mathrm{L}], \mathrm{T}$ is the surface tension between the fluid and the capillary $\left[\mathrm{MT}^{-2}\right], \beta$ is the contact angle between the fluid and the tube [rad], $\mu$ is the viscosity of the fluid $\left[\mathrm{ML}^{-1} \mathrm{~T}^{-3}\right]$ and, $\rho_{\mathrm{w}}$ is the density of the fluid $\left[\mathrm{ML}^{-3}\right]$.

Eq. (1) can be solved analytically considering different flow regimes. For small times (initial regime) has been $\mathrm{z}=0$ implicated in $\mathrm{dz} / \mathrm{dt}=0$ at Eq. (1) resulting in $\mathrm{z}(\mathrm{t}) \sim \mathrm{t}^{2}$. At a viscous regime, the inertial effects become negligible. At early viscous regime, $\mathrm{dz} / \mathrm{dt}$ is independent of $\mathrm{g}$. There are only viscous pressure loss contributions making the terms inertial and hydrostatic pressure negligible in Eq. (1). This condition results in the classic solution obtained by Lucas (1918) [12] and Washburn (1921) [13] at which the capillary height is $z(t) \sim t^{1 / 2}$. The late viscous regime has $z(t)=h_{c, \text { max }}$, or capillary equilibrium height, i.e., for infinite times, a steady state is reached where the hydrostatic pressure balances the capillary pressure (Zhmud et al. 2000 [14], Fries and Dreyer [11]).

Gois et al. [10] solved Eq.(1), considering that the flow paths in porous media are generally tortuous (Cai et al. 2010 [15]) and that the tests of capillary rise are longlasting and the data converge to $t \rightarrow \infty$.

\section{Materials and Methods}

\subsection{Porous medium}

The experiments were performed with civil construction sand that was selected using all material passing through the $2 \mathrm{~mm}$ sieve (N10). The physical and hydraulic characteristics are listed in Table 1. The geotechnical tests were performed according to the classification, followed by the American Society for Testing and Materials (ASTM) guideline. The material porosity was assumed to be equal to the saturated volumetric water content $(\mathrm{n}=$ 0.44 ), and the void ratio value $\mathrm{e}=0.78$.

Table 1. Material geotechnical characterization

\begin{tabular}{c|c|c}
\hline Test & \multicolumn{2}{|c}{ Property } \\
\hline \multirow{2}{*}{ Granulometry } & $\mathrm{D} 10(\mathrm{~mm})$ & 0.25 \\
\cline { 2 - 3 } & $\mathrm{D} 20(\mathrm{~mm})$ & 0.45 \\
\cline { 2 - 3 } & $\mathrm{D} 30(\mathrm{~mm})$ & 0.85 \\
\hline $\begin{array}{c}\text { Grain Specific } \\
\text { Mass }\end{array}$ & $\mathrm{G}_{\mathrm{S}}$ & 2.70 \\
\hline $\begin{array}{c}\text { Atterberg } \\
\text { Limits }\end{array}$ & $\mathrm{LL}(\%)$ & $\mathrm{NA}^{*}$ \\
\cline { 2 - 3 } & $\mathrm{IP}(\%)$ & $\mathrm{NA}^{*}$ \\
\hline $\begin{array}{c}\text { Saturated } \\
\text { Permeability }\end{array}$ & $\mathrm{k}_{\mathrm{s}}\left(\mathrm{m}^{-1} \mathrm{~s}^{-1}\right)$ & $2.40 \times 10^{-4}$ \\
\hline $\begin{array}{c}\text { Volumetric } \\
\text { Water Content }\end{array}$ & $\theta_{\mathrm{s}}\left(\mathrm{m}^{3} \cdot \mathrm{m}^{-3}\right)$ & 0.44 \\
\cline { 2 - 3 } & $\theta_{\mathrm{r}}\left(\mathrm{m}^{3} \cdot \mathrm{m}^{-3}\right)$ & 0.01 \\
\multirow{2}{|c}{$\begin{array}{l}\text { Aplicable } \\
\text { Not Appling }\end{array}$}
\end{tabular}

For the columns' assembly, a mechanical agitator was used, making it possible to compact the soil, resulting in a specific mass of approximately $1550 \mathrm{~kg} \cdot \mathrm{m}^{-3}$. In sequence, the columns were placed on a geotextile and a polyvinyl chloride (PVC) base drilled on the sides and filled with rocks of different sizes inside a plastic tank. This procedure was adopted to simulate semi-infinite column experiments.

The water's electrical conductivity used $4.30 \times 10^{-3} \mathrm{~S} \cdot \mathrm{m}^{-1}$ at $21^{\circ} \mathrm{C}$. The water level in the tank was kept constant throughout the experiment.

\subsection{Experimental Set Up - Fundamentals, Data Acquisition, and Pre-Processing}

\subsubsection{Column 1- Spontaneous Potential}

The self-potential (SP) method is based on the fact that it is possible to measure a difference in electrical potential between two electrodes located on the surface of the terrain (or interior of wells) generated by electric current sources existing on the terrestrial substrate. These sources have their origin in natural processes that involve coupled flows, in which gradients of a primary potential (pressure, temperature, or electrochemical) establish a flow of electrical charges underground (Sill 1983 [16]).

Thus, there is a polarization of the medium and a measurable potential on the surface. According to the coupled flow mechanism, the electrical potential supports a secondary current flow that persists as long as the primary flow is in effect (Sill [16]).

The electrical potentials in coupled flow processes are named according to the primary flow (electrokinetic, electrochemical, and redox reactions). Potentials of electrokinetic origin (streaming potential) are generated when the primary flow results from a pressure gradient that sets the groundwater in motion.

Theoretically, when water moves through a saturated capillary system by laminar flow, the electric potential difference $\Delta \mathrm{V}\left[\mathrm{ML}^{2} \mathrm{~T}^{-3} \mathrm{~A}^{-1}\right]$ is defined by the relation $\Delta \mathrm{V}$ $=\mathrm{C} \Delta \mathrm{P}$, where $\Delta \mathrm{P}$ is the pressure gradient $\left[\mathrm{ML}^{-1} \mathrm{~T}^{-2}\right]$. The constant $\mathrm{C}$, called the coupling constant, or streaming potential coefficient represents the fluid's physicochemical characteristics (Eq.(2)).

$$
\Delta \mathrm{V}=\frac{\xi_{\varepsilon \Delta \mathrm{P}}}{\mu \sigma}
$$

where, $\xi$ represents a potential difference in the double layer at the solid-liquid interface $\left[\mathrm{ML}^{2} \mathrm{~T}^{-3} \mathrm{~A}^{-1}\right] ; \varepsilon, \mu, \sigma$ are: dielectric permittivity $\left[\mathrm{ML}^{-3} \mathrm{~T}^{4} \mathrm{~A}^{2}\right]$, viscosity $\left[\mathrm{ML}^{-1} \mathrm{~T}^{-3}\right]$ and electrical conductivity $\left[\mathrm{M}^{-1} \mathrm{~L}^{-3} \mathrm{~T}^{3} \mathrm{~A}^{2}\right]$ respectively, of the electrolyte-water.

Laboratory experiments were conducted because the water, when ascending through the capillaries formed by the soil's solid particles, carries the cations. At the same time, the anions remain fixed in the capillaries generating a potential difference (DDP) that can be mapped to infer the hydraulic characteristics of the medium (Revil et al. [1], Franzoni \& Bandini 2012 [4], Revil et al. [6], Oliveti \& Cardarelli [8]).

The column was instrumented with silver electrodes, 0.25 $\times 10^{-1} \mathrm{~m}$ long with a sodium chloride coating were used to avoid electrode polarization problems (Telford et al. 1990 
[17]). These electrodes were arranged longitudinally simulating the potential technique, that is, the electrode 1 reference fixed at $0.10 \mathrm{~m}$ from the base of the column connected to the negative terminal and the other electrodes $(2,3,4,5,6$ and 7 to $0.15 \mathrm{~m}, 0.20 \mathrm{~m}, 0.25 \mathrm{~m}$, $0.30 \mathrm{~m}, 0.35 \mathrm{~m}$ and $0.40 \mathrm{~m}$ from the base of the column, respectively) were connected in sequence to the positive terminal of a bench multimeter with high input impedance (Fig. 1).

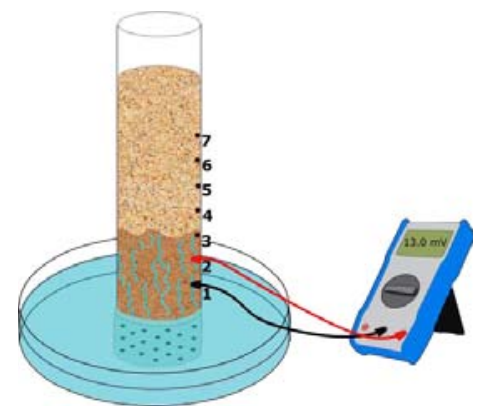

Fig. 1. Capillary rise monitoring with spontaneous potential method - column 1

This multimeter recorded the electrical potential associated with the electrokinetic from the pressure gradient caused by water movement in column 1 (Eq.(2) ).

It was assumed that by eliminating the noise of different natures, using non-polarizable electrodes, and in the absence of strong oxidoreduction processes, the main contributor to self-potential anomalies corresponds to an electrokinetic conversion of the capillary rise.

SP measurements were recorded over time, and as the moisture front progressed. The data were pre-processed to eliminate spurious data and corrected by a factor of 0.9 $\mathrm{mV}$ obtained when measuring the DDP of an electrode. In the sequence, SP measurements' profiles were constructed over time (Fig. 5), and the correlation of SP anomalies with capillary height was made (Fig. 6) - the profiles are presented in section 3 up to the pair of electrodes 1-5.

\subsubsection{Column 2 - Low-cost Soil Moisture Sensor}

The moisture sensor measures a potential difference in the medium associated with a measure of electrical resistivity (ER). The ER of a soil sample is governed mainly by the fluid's nature, the proportion of voids within the sample, distribution of particles, tortuosity, the concentration of salts in the fluid, and degree of saturation (Keller and Frischknecht 1966 [18]).

Several studies have used Archie's law to relate petrophysical parameters to the electrical conductivity (EC) or ER of rocks and soils. Among them, Shah and Singh (2005) [19] carried out studies related to the volumetric water content, fluid electrical conductivity, petrophysical parameters (characteristics of soil particle size and shape of the grains or pores associated with tortuosity/interconnectivity) the electrical conductivity of an unsaturated medium.

Therefore, it is possible to measure the soil's electrical resistivity and relate it to the state of soil moisture (Gois et al. [9]), i.e., the higher the soil moisture, the lower its electrical resistivity.

The monitoring system in column 2 used the YL-69 sensor as its main element. This sensor is of the resistive type, composed of two parts, an electronic plate with a potentiometer and a probe with two rods with a metallic tape (simulating two electrodes), which inject a current in the medium and measure a potential difference that is associated the electrical resistivity of that medium in conducting the electric current.

The Arduino Mega microcontroller translates such electrical resistivity into a dimensionless measurement in bits (analog output between 0 and 1023) with a resolution of $\simeq 0.00488759 \mathrm{~V}$ per unit. Thus, the higher the soil moisture, the lower its ER, and the lower the measured bit.

To record the data collected during the experiments were used: an Arduino Mega microcontroller board (ATMEGA 2560); SD Card Module with 8 Gb Micro Sd Memory Card for data storage; DS1307 Real Time Clock (RTC) module for recording data with date and time; $1200 \mathrm{Ohm}$ resistors; battery; light-emitting diodes (LED); and the YL-69 sensors, all instrumentation cost approximately US $\$ 67.50$. The instrumentation code for the column is available at https://github.com/NicolasOhofugi/C-digoSensor-de-Umidade-YL-69 (Ohofugi et al. 2019 [20]).

The column was instrumented with moisture sensors arranged longitudinally at $0.10 \mathrm{~m}-\mathrm{S} 1 ; 0.20 \mathrm{~m}-\mathrm{S} 2 ; 0.30$ $\mathrm{m}-\mathrm{S} 3$ and $0.40 \mathrm{~m}-\mathrm{S} 4$ from the column's base (Fig. 2).

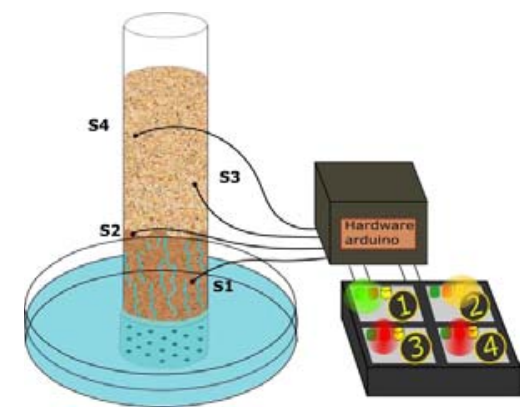

Fig. 2. Capillary rise monitoring with low-cost moisture sensor - column 2.

The data were acquired every 5 seconds, making it possible to build monitoring profiles with high sampling for each sensor over time (Fig. 7) and correlate the variations of the measurements in bits to the capillary's transient and stationary behavior rise phenomenon. Finally, the volumetric water profiles (Fig. 8) are obtained through the petrophysical relationship obtained by Gois et al. [10], which relates to the petrophysical characteristics of the medium and the measurements in bits (Eq.(3)).

$$
\theta(\text { Bits })=\left(\frac{a e^{j B i t s}}{\tau n^{-m+p} E R_{w}}\right)^{-1 / p}
$$

where $a$ and $j$ are dimensionless fit parameters for sandy soil, $\mathrm{ER}_{\mathrm{w}}$-water electrical resistivity, $\tau$-tortuosity, $\mathrm{n}$ porosity, m-cementation exponent, $\mathrm{p}$-saturation exponent. The profiles for the 4 monitored horizons are presented in section 3 (Fig. 7, and Fig. 8). 


\subsubsection{Column 3 - Electrical Resistivity}

The physical principles that were used for the electrical resistivity measurements in column 3 are the same as those used in column 2 , so it was decided to monitor the $0.15 \mathrm{~m}$ horizon for more than 4 hours to validate the electrical resistivity changes and for consequently, the volumetric water content in the transient region of the phenomenon.

The column was instrumented with 16 silver electrodes arranged radially (Fig. 3) with a sodium chloride coating, with the same specification as those used in column 1 to avoid the problem of electrode polarization. The readings were performed by the Syscal Pro 72 resistivimeter (Iris Instruments $\left.{ }^{\circledR}\right)$.

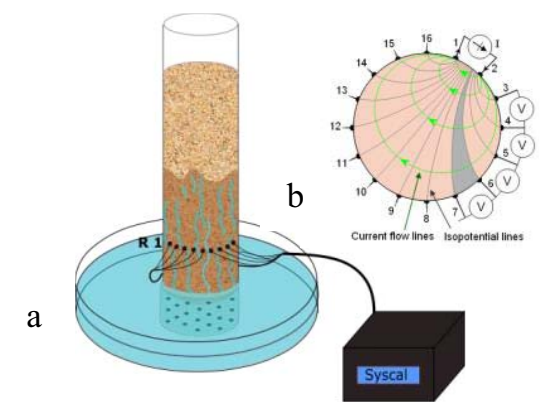

Fig. 3. Monitoring of capillary rise - column 3 a) Instrumentation with Syscal Pro 72 and 16 non-polarizable silver electrodes. b) Data acquisition with dipole-dipole arrangement - adapted from Visentin \& Suzuki 2015 [21].

The dipole-dipole electrode array was used in which an electric current is injected, and a potential difference is measured. With this radial arrangement, 16 pairs of current injection were obtained. For each pair of current injection, 13 pairs of potential measurements along the ring, this methodology is used in tomographic studies. It allows for mapping the spatial distribution of electrical conductivity.

With the measurements made on the ring, the potential difference, the electrical current, and the average electrical resistance for the 16 pairs of current injection were calculated. Afterward, the apparent electrical resistivity $\left(\mathrm{ER}_{\mathrm{a}}\right)$ Eq.(4) was calculated.

$$
\mathrm{ER}_{\mathrm{a}}=\mathrm{kER}_{\mathrm{av}}
$$

where $\mathrm{k}=0.521 \mathrm{~m}-$ generalized geometric factor obtained according to the methodology of Marescot et al. (2006) [22] and $\mathrm{ER}_{\mathrm{av}}$ average electrical resistance in $\Omega$.

Using Eq. (5), which relates the petrophysical parameters of the soil and fluid with the $\mathrm{ER}_{\mathrm{a}}$, it was possible to monitor the volumetric water content in the horizon of interest (Gois et al. [10]).

$$
\theta\left(\mathrm{ER}_{\mathrm{a}}\right)=\left(\frac{\mathrm{ER}_{\mathrm{a}}}{\tau \mathrm{n}^{-\mathrm{m}+\mathrm{p}} \mathrm{ER}_{\mathrm{w}}}\right)^{-1 / \mathrm{p}}
$$

The profiles of the time-lapse monitoring of the electrical potential (Fig. 9) and the volumetric water content (Fig. 10) are presented in section 3 .

\subsubsection{Column 4 - Ground Penetrating Radar (GPR)}

GPR is a geophysical method extensively used to map subsurface structures, with applications in the most diverse areas (Jol 2009 [3]). The method uses the physical principles of electromagnetic wave propagation. GPR uses radio waves in a frequency spectrum from $\mathrm{MHz}$ to $\mathrm{GHz}$, which gives it the characteristic of an investigative method of very high resolution.

In bi-static mode, the transmitting antenna radiates a highfrequency electromagnetic pulse that propagates through the subsurface. The receiving antenna captures the signal originally emitted by the transmitter from different ways. This spherical wave radiation process gives rise to waves that propagate directly from the transmitting antenna to the receiver through the ground and air. The waves that reach the receiving antenna after being refracted and reflected in the media interfaces with different electrical properties.

Considering only the electromagnetic wave's reflection events, the reflection coefficients are obtained from the electromagnetic impedance. The amplitude of the GPR's reflection coefficient is the ratio of the electromagnetic impedances of each medium (Eq.(6)) that can be expressed by their dielectric permissivities or speeds.

$$
\mathrm{r}_{\mathrm{GPR}}=\frac{\sqrt{\varepsilon_{1}}-\sqrt{\varepsilon_{2}}}{\sqrt{\varepsilon_{1}}+\sqrt{\varepsilon_{2}}}=\frac{\mathrm{v}_{2}-\mathrm{v}_{1}}{\mathrm{v}_{2}+\mathrm{v}_{1}}
$$

where: $\varepsilon_{1}, \varepsilon_{2}, \mathrm{v}_{1}$, and $\mathrm{v}_{2}$ are dielectric permittivities [ML $\left.{ }^{3} \mathrm{~T}^{4} \mathrm{~A}^{2}\right]$, and velocities $\left[\mathrm{LT}^{-1}\right]$ of the reflected wave from medium 1 and 2 , respectively.

The column was instrumented with a $2.6 \mathrm{GHz}$ shielded antenna (Geophysical Survey Systems - GSSI ${ }^{\circledR}$ ) operating in bi-static mode - the separation between 40 $\mathrm{mm}$ antennas. The antenna was positioned at the top of the column, and the data was acquired by simulating a common offset profile (Fig. 4).

The data were pre-processed in REFLEXW 7.2.3 (Sandmeier 2014 [23]) following the sequence: correction of zero time, concatenation of the files, analysis of the amplitude and frequency spectra, picking of the events corresponding to the reflected wave, to export of the picking file and, calculation of speeds using heights monitored directly on the column. The velocities were calculated for each event $v=2 h / t$, where $h$ is the capillary height visually monitored in the column, and $t$ is the double time of the reflection event.

The dielectric permittivity of that horizon was calculated using the relation $\varepsilon=(\mathrm{c} / \mathrm{v})^{2}$ where c-velocity of the electromagnetic wave in the air $(0.3 \mathrm{~m} / \mathrm{ns})$ and the velocity of the identified event. 


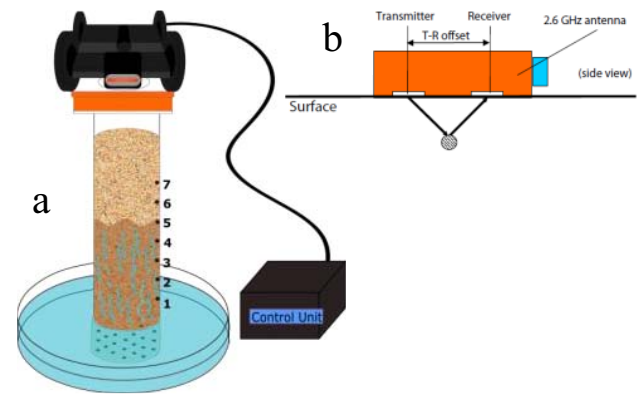

Fig. 4. Capillary rise monitoring with GPR. a) $2.6 \mathrm{GHz}$ shielded antenna, and b) Side view of the reflected wave antenna (from GSSI ${ })$.

Finally, using the relationship of Topp et al. (1980) [24], the volumetric water content was determined from the dielectric permittivity measures (Eq.(7)).

$\theta=-5.3 \times 10^{-2}+2.92 \times 10^{-2} \varepsilon-5.5 \times 10^{-4} \varepsilon^{2}+4.3 \times 10^{-6} \varepsilon^{3}$

The identification of the top and bottom of the column and the transient monitoring of the reflector corresponding to capillary rise via the change in electromagnetic characteristics (dielectric permittivity) are presented in section 3 (Fig. 11 and Fig. 12).

\section{Experimental Results and Discussions}

The capillary rise of water was investigated in the four soil columns using geophysical methodologies with different physical principles, but all sensitive to water present in the environment.

In Column 1, monitoring over time of the electrical potential during the transient and stationary regimes associated with the pressure gradient caused by the capillary water rise was used. According to Eq.(2), considering only variations in hydrostatic pressure at the ends of the soil's capillaries, the lower the hydrostatic pressure, the more significant the potential difference recorded. Negative and positive anomalies were mapped in the monitoring horizons throughout the phenomenon (Fig. 5).

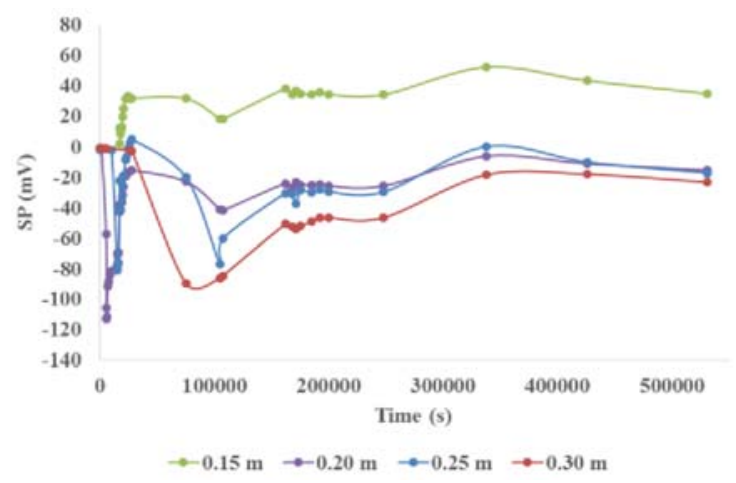

Fig. 5. Time-lapse monitoring of SP in electrode pairs 1-2, 1-3, $1-4$ and $1-5$ which corresponds to the heights of $0.15 \mathrm{~m}, 0.20 \mathrm{~m}$, $0.25 \mathrm{~m}, 0.30 \mathrm{~m}$, respectively - column 1 .
In the horizons of $0.20 \mathrm{~m}$ and $0.30 \mathrm{~m}$, negative anomalies of great amplitude were observed from 5520 seconds until 18240 seconds, which corresponds to the region of an early viscous regime where the capillary height has a $t^{1 / 2}$. Washburn regime. Subsequently, there is another region of negative anomalies for the horizons $0.20 \mathrm{~m}, 0.25 \mathrm{~m}$, and $0.30 \mathrm{~m}$ from 105120 seconds when the viscous regime starts with the predominance of the gravitational effect and the capillary height is directly related to the maximum height that the moisture front has reached (Fig. 5).

It was observed that even when the moisture front reached its maximum expected height after 105120 seconds, the

SP measurements still showed oscillations, and after 338160 seconds, they started to stabilize (Fig. 5), registering positive and negative anomalies of the order of $35 \mathrm{mV}$ a $-24 \mathrm{mV}$, respectively.

A correlation was made between visual capillary height monitoring and SP anomalies (Fig. 6). The negative anomaly of greater amplitude $112.09 \mathrm{mV}$ was recorded in the pair of electrodes 1-3, which was related to the moment the capillary height reached $0.20 \mathrm{~m}$. Subsequently, in electrodes $1-5$, a negative anomaly with an amplitude of $89.6 \mathrm{mV}$ was recorded when the capillary height was approximately $0.29 \mathrm{~m}$.

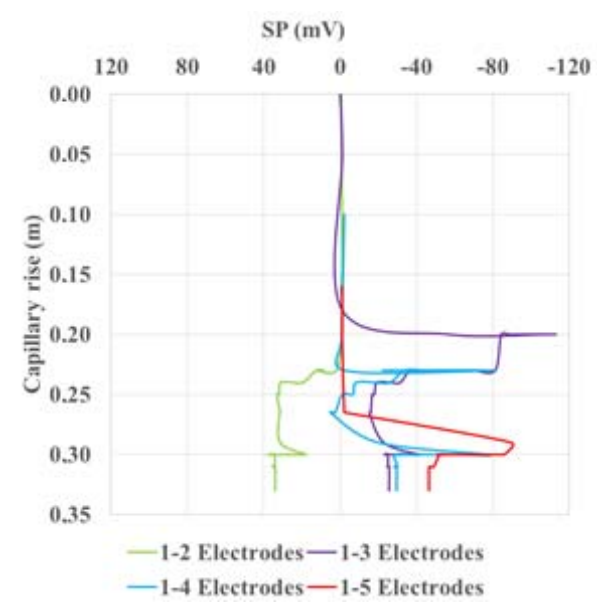

Fig. 6. Correlation of SP anomalies measured in electrode pairs 1-2,1-3,1-4 and 1-5 with capillary rise monitoring - column 1.

Column 2 used low-cost moisture sensors every $0.10 \mathrm{~m}$ to monitor the transient behavior of the phenomenon and monitor it until it reaches the steady-state condition.

According to the sensor calibration performed by Gois et al. [9], the initial instant that the moisture sensor registered bits less than or equal to 900 was assumed to be the moment when the water percolated the horizon where the sensor was measuring. The S1 that was located closest to the water level registered in 22625 seconds abrupt variations in its measurements, going from 1023 bits to 898 bits. The S2, in turn, recorded the reading of 899 bits from 24440 seconds. The S3 took 119055 seconds to register the 900-bit reading, and the $\mathrm{S} 4$ showed no variations that indicated the arrival of the moisture front on that sensor, keeping its readings between 1023 bits and 1022 bits throughout the test (Fig. 7). 


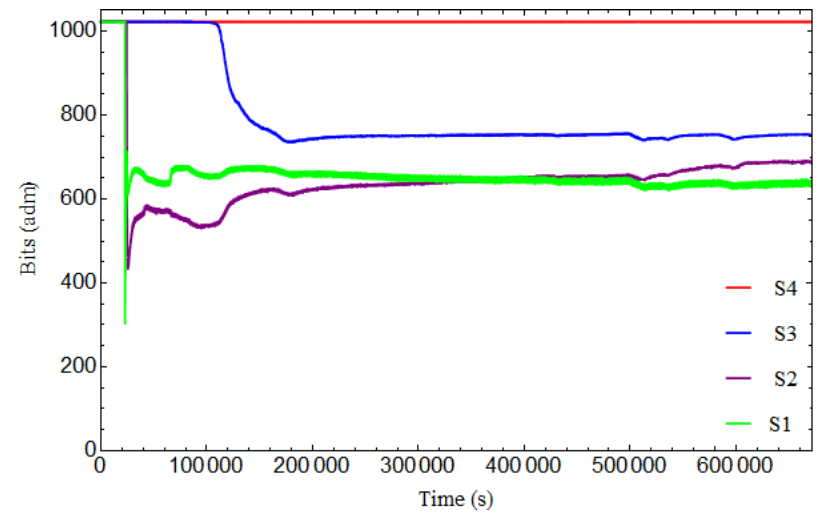

Fig. 7. Time-lapse monitoring of moisture sensors (bits) at 0.10 $\mathrm{m}-\mathrm{S} 1 ; 0.20 \mathrm{~m}-\mathrm{S} 2 ; 0.30 \mathrm{~m}-\mathrm{S} 3$ and $0.40 \mathrm{~m}-\mathrm{S} 4$ from the base of column 2 .

The moisture front reached the region of an early viscous regime where the capillary height has a $\mathrm{t}^{1 / 2}$-Washburn regime, which corresponds to oscillations in the bit values for the horizons of S1 at $0.10 \mathrm{~m}$ and $\mathrm{S} 2$ at $0.20 \mathrm{~m}$ up to 180000 seconds. It is observed that up to 320000 seconds, S2 has bits smaller than S1 (Fig. 7).

The S3 at $0.30 \mathrm{~m}$ has a more stable behavior with reductions in the bit values when the phenomenon starts to tend towards the stationary regime and then remains constant until the end of the monitoring.

It can be seen in Fig. 7 that only after 480000 seconds that the sensors maintain the bit behavior for $\mathrm{S} 1<\mathrm{S} 2<\mathrm{S} 3<\mathrm{S} 4$, being so from that moment on by this monitoring methodology that it is assumed that the medium has reached balance condition (Fig. 7).

Using the semi-empirical formulation (Eq.(3)) to convert bits into volumetric water content, it was possible to observe the evolution of the volumetric water content over time (Fig. 8). The horizon at $0.10 \mathrm{~m}$ from the column's base has the highest value of volumetric water content at approximately $52 \%$, followed by the horizon of $0.20 \mathrm{~m}$ with $34 \%$ and the horizon of $0.30 \mathrm{~m}$ with $13 \%$, all in the region of early viscous (Fig. 8).

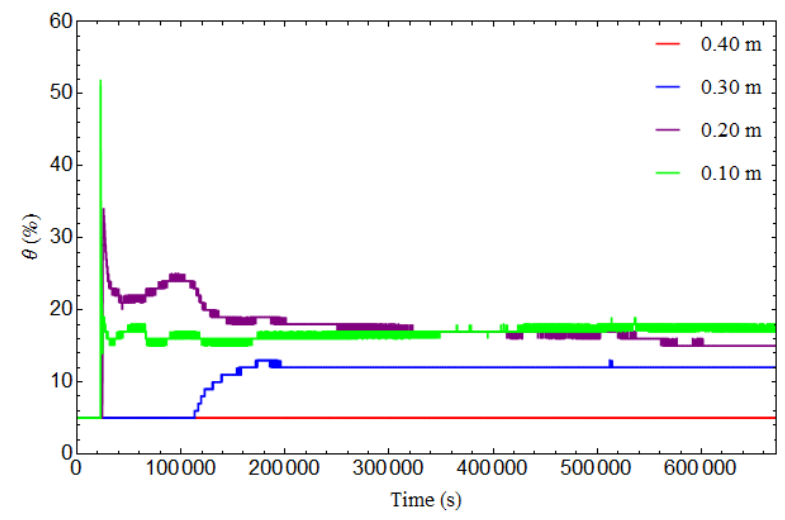

Fig. 8. Time-lapse monitoring of volumetric water content at different heights of the 2 soil column. Parameters from Gois et al. 2020 [10]: $\mathrm{a}=250, \mathrm{j}=2.2 \times 10^{-3}, \mathrm{ER}_{\mathrm{w}}=232.56 \Omega \mathrm{m}, \tau=1.36, \mathrm{n}$ $=0.44, \mathrm{~m}=0.65$, and, $\mathrm{p}=0.68$.
After 200000 seconds, the volumetric water content remained constant in the horizons of $0.10 \mathrm{~m}$ and $0.30 \mathrm{~m}$, and only after approximately 400000 seconds that the horizon of $0.20 \mathrm{~m}$ has a volumetric water content lower than the horizon of $0.10 \mathrm{~m}$ until the end of the monitoring, being thus, it is evident that even when the moisture front reaches its maximum capillary height of $0.30 \mathrm{~m}$ in approximately 105000 seconds, the equilibrium condition of the phenomenon is only reached after 400000 seconds when the moisture content remains constant in each horizon with $\theta_{\mathrm{h}=0.10 \mathrm{~m}}>\theta_{\mathrm{h}=0.20 \mathrm{~m}}>\theta_{\mathrm{h}=0.30 \mathrm{~m}}>\theta_{\mathrm{h}=0.40 \mathrm{~m}}$ (Fig. 8).

In Column 3, no electric current circulation nor electrical potential was detected in the initial instants, 3864 seconds after water enters the system. The electrical potential begins to vary for the different pairs of electrodes (Fig. 9). It is observed that a pair of specific electrodes record electrical potentials ranging from $7061 \mathrm{mV}$ to $4530 \mathrm{mV}$. This is related to the fact that there are regions along the ring where water percolation takes preferential paths.

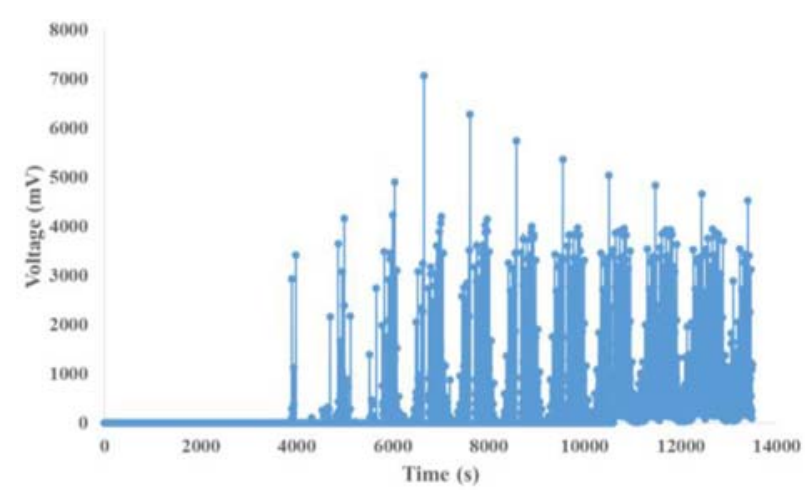

Fig. 9. Time-lapse monitoring of the voltage measured at the different pairs of electrodes - column 3

The transient moisture profile (Fig. 10) of the $0.15 \mathrm{~m}$ horizon was constructed using Eq.(5), with maximum values of $74.75 \%$ and $48.50 \%$.

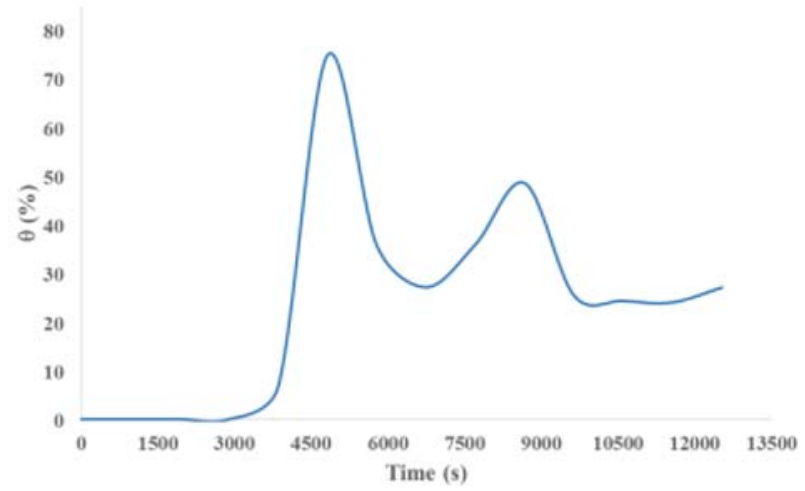

Fig. 10. Time-lapse monitoring of the ER at a height of $0.15 \mathrm{~m}$ - column 3. Parameters from Gois et al. [10]: $\mathrm{ER}_{\mathrm{w}}=232.56 \Omega \mathrm{m}$, $\tau=1.36, \mathrm{n}=0.44, \mathrm{~m}=0.65$, and $\mathrm{p}=0.68$

In column 4, GPR was used because it is a high-resolution geophysical method to monitor for 1.17 hours as a phenomenon developed in the transient region. In the radargram of Fig. 11, the reflectors corresponding to the 
top and bottom of the column and two reflectors that are associated with the advance of the moisture front can be seen.

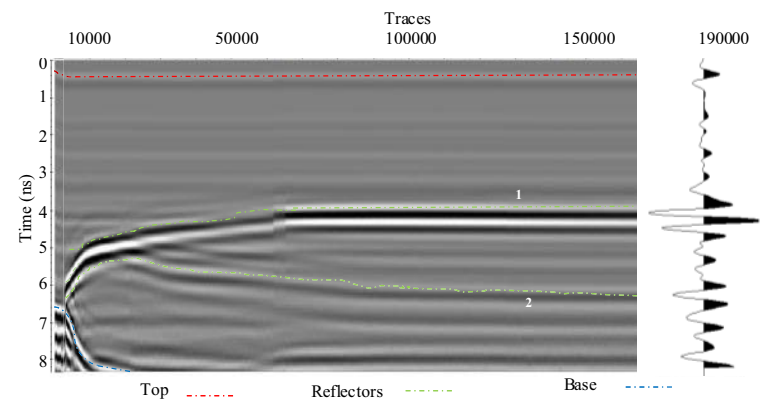

Fig. 11. Radargram of capillary rise time-lapse monitoring column 4.

The top and bottom reflectors of the column have been identified $\mathrm{v}_{\text {Top }}=0.27 \mathrm{~m} . \mathrm{ns}^{-1}, \varepsilon_{\text {Top }}=1.27, \theta_{\text {Top }}=\sim 0 \%$, and $\mathrm{v}_{\text {Base }}=0.14 \mathrm{~m} . \mathrm{ns}^{-1}, \varepsilon_{\text {Base }}=4.34, \theta_{\text {Base }}=6.37 \%$.

As the water started to ascend from trace 2495, it is observed that reflectors appear (Fig. 11). Analyzing reflector 1, when trace 61855 was reached, the apparent height of the moisture front was $0.18 \mathrm{~m}$, which corresponds to a $\mathrm{V}_{\mathrm{R} 1(0.18 \mathrm{~m})}=0.09 \mathrm{~m} \cdot \mathrm{ns}^{-1}, \varepsilon_{\mathrm{R} 1(0.18 \mathrm{~m})}=10.84 \mathrm{e}$ $\theta_{\mathrm{R} 1(0.18 \mathrm{~m})}=20.43 \%$. In the sequence, when the apparent moisture front was $0.20 \mathrm{~m}$ and $0.22 \mathrm{~m}$, the corresponding traces were 127391 and 192920 , respectively. Therefore, the values resulted in $\mathrm{V}_{\mathrm{R} 1(0.20 \mathrm{~m})}=0.10 \mathrm{~m} \cdot \mathrm{ns}^{-1}, \varepsilon_{\mathrm{R} 1(0.20}$ $\mathrm{m})=8.86, \theta_{\mathrm{R} 1(0.20 \mathrm{~m})}=16.55 \%$, and $\mathrm{v}_{\mathrm{R} 1(0.22 \mathrm{~m})}=0.11 \mathrm{~m}^{\mathrm{nn}} \mathrm{s}^{-1}$, $\varepsilon_{\mathrm{R} 1(0.22 \mathrm{~m})}=7.19, \theta_{\mathrm{R} 1(0.22 \mathrm{~m})}=13.01 \%$.

When analyzing the values of the amplitude attribute of reflector 1 (Fig. 12), an oscillatory tendency is observed up to the number 68012, which corresponds to approximately $0.20 \mathrm{~m}$ at the capillary height, and later the amplitude starts to increase steadily until the number line 192927 which corresponds to the height of $0.22 \mathrm{~m}$.

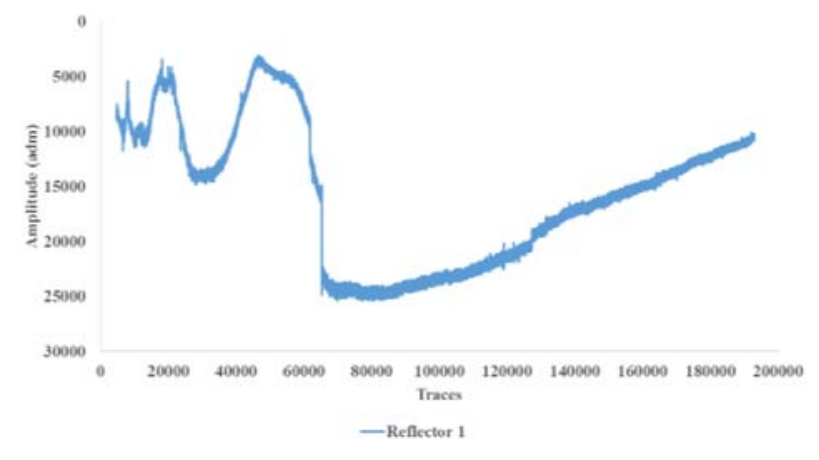

Fig. 12. 1.17-hour monitoring of the amplitude attribute during capillary rise - column 4.

\section{Conclusions}

The instrumentation assembled proved to be satisfactory. It is possible to monitor the capillary rise at the time with different methodologies. The maximum capillary height of $0.30 \mathrm{~m}$ predicted by the classic models was reached after 29.2 hours. The monitoring lasted $187 \mathrm{~h}$, and it was found that the maximum capillary height remains constant for a long time.

The SP method shows the dynamics of the phenomenon of capillary rise very well. For regions where there is no influence of the gravitational force, which corresponds to a capillary height less than $0.30 \mathrm{~m}$, the anomalies are predominantly negative, except for the measurements of the pair of electrodes 1-2, later when the gravitational effect dominates the maximum capillary height $(0.30 \mathrm{~m})$ is reached. It was observed that the SP measurements over time still took some time to stabilize after the moisture front has reached the maximum capillary height. (column 1).

Continuous monitoring with low-cost sensors (column 2) showed the phenomenon's dynamics for regions with the transient and stationary regime. The sensors were able to map the evolution of the phenomenon; the 0.20 m horizon had a peculiar behavior compared to the other's horizons to reach the equilibrium state, which was also observed in the SP data.

The monitoring of the electrical potential / electrical resistivity (column 3) showed that the water that ascends the medium takes preferential paths and that after detecting values of electrical current and electrical potential in that horizon, the minimum and maximum volumetric water content values were $6.69 \%$ and $74.75 \%$, respectively.

Finally, in column 4, the amplitude of the reflected GPR signal was monitored to show that the electromagnetic wave's amplitude varies as the moisture front ascends. It was also possible to correlate the electromagnetic wave speed measurements, permissiveness dielectric, and moisture content over time. As seen in columns 2 and 3, the initial moisture content is high, and as the moisture front increases, the values decrease.

With the different indirect measures that were taken, it was evident that the region of the transient regime has an oscillatory behavior for the analyzed physical parameters and that after reaching the maximum capillary height, there are still variations in the volumetric water content and the rising moisture front takes time to reach its equilibrium state.

\section{Acknowledgments}

This study was financed in part by the Coordination for the Improvement of Higher Education Personnel - Brasil (CAPES) - Finance Code 001. The authors also acknowledge the support of the National Council for Scientific and Technological Development (CNPq Grant 304721/2017-4 and 435962/2018-3), the Foundation for Research Support of the Federal District (FAPDF) (Projects 0193.002014/2017-68 and 0193.001563/2017), 
the CEB Geração S.A. (PD-05160-1904/2019), and the University of Brasília, including the Laboratory of the Physics and Chemistry of Soils of the School of Agronomy, the Laboratory of Applied Geophysics, and the Laboratory of Geochemistry of the Institute of Geosciences.

\section{References}

1. A. Revil, V. Naudet, J. Nouzaret, \& M. Pessel. (2003). Principles of electrography applied to selfpotential electrokinetic sources and hydrogeological applications. Water Resources Research, 39(5).

2. O. Loeffler, \& M. Bano, (2004). Ground penetrating radar measurements in a controlled vadose zone: Influence of the water content. Vadose Zone Journal, 3(4), 1082-1092.

3. H. M. Jol, (Ed.). (2009). Ground penetrating radar theory and applications. Elsevier. https://doi.org/10.1016/B978-0-444-533487.X0001-4

4. E. Franzoni, \& S. Bandini. (2012). Spontaneous electrical effects in masonry affected by capillary water rise: the role of salts. Construction and Building Materials, 35, 642-646.

5. P. Klenk, S. Jaumann, \& K. Roth. (2015). Quantitative high-resolution observations of soil water dynamics in a complicated architecture using time-lapse ground-penetrating radar. Hydrology and Earth System Sciences, 19(3), 1125.

6. A. Revil, A. S. Ahmed, \& A. Jardani. (2017). Selfpotential: A non-intrusive ground water flow sensor.Journal of Environmental and Engineering Geophysics,22(3), 235-247.

7. X. Liu, J. Chen, X. Cui, Q. Liu, X. Cao, \& X. Chen, (2017). Measurement of soil water content using ground-penetrating radar: a review of current methods. International Journal of Digital Earth, 12(1), 95-118.

8. I. Oliveti, \& E. Cardarelli. (2019). Self-Potential Data inversion for environmental and hydrogeological investigations. Pure and Applied Geophysics,176(8), 3607-3628.

9. M. S. Gois, A. L. B. Cavalcante. (2019). Estudo Quantitativo das Concentrações de Soluto e Níveis de Saturação de Fluidos a Partir de Estudos Hidrogeofísicos na Zona Não Saturada. Relatório Técnico Anual de Pós-Doutorado; PPG em Geotecnia, Dep. de Eng. C. e A., Universidade de Brasília (in portuguese).

10. M. S. Gois, N. O. Guimaraes, K. R. C. B. Costa, A. L. B. Cavalcante. (2020). Prediction of hydraulic properties and capillary height of granular soils during time-lapse monitoring of capillary rise, Report No.01, Department of Civil and Environmental Engineering, University of Brasilia, Brazil.
11. N. Fries, \& M. Dreyer. (2008). An analytic solution of capillary rise restrained by gravity. Journal of colloid and interface science, 320(1), 259-263.

12. R. Lucas. (1918). Rate of capillary ascension of liquids (Ueber das Zeitgesetz des kapillaren Aufstiegs von Flussigkeiten). Kollid-Zeitschrift. V. 23, N. 1, pp. 15-22.

13. E. W. Washburn. (1921). The dynamics of capillary flow. Physical review, 17(3), 273.

14. B. V. Zhmud, F. Tiberg, K. Hallstensson. (2000). Dynamics of capillary rise.Journal of colloid and interface science, 228(2), 263-269.

15. C. Jian-Chao, Y. Bo-Ming, M. Mao-Fei, \& L. Liang, (2010). Capillary rise in a single tortuous capillary. Chinese Physics Letters, 27(5), 054701.

16. W. R. Sill. (1983). Self-potential modeling from primary flows. Geophysics 48, 76-86.

17. W. M. Telford, L. P. Geldart, \& R. E. Sheriff. (1990). Applied. Geophysics (Vol. 1). Cambridge University Press.

18. G. V. Keller, \& F. C. Frischknecht. (1966). Electrical methods in geophysical prospecting. Oxford: Pergamon Press.

19. P. H. Shah, \& D. N. Singh. 2005. Generalized Archie's law for estimation of soil electrical conductivity. J. ASTM Int. 2 (5), 1-20.

20. N. G. Ohofugi, M. S. Gois, K. R. C. B. da Costa, A. L. B. Cavalcante. 2019. Instrumentação de Baixo Custo em Coluna de Solo para Monitoramento TimeLapse da Ascensão Capilar. In: $5^{\circ}$ Simpósio de Prática de Engenharia Geotécnica na Região Centro Oeste (GEOCENTRO 2019).

21. F. Visentin, \& K. Suzuki, (2015). Deformable sensors for soft robot by electrical impedance tomography. In 2015 IEEE International Conference on Robotics and Biomimetics (ROBIO) (pp. 10061011). IEEE.

22. L. Marescot, S. Rigobert, S. P. Lopes, R. Lagabrielle, \& D. Chapellier (2006). A general approach for DC apparent resistivity evaluation on arbitrarily shaped 3D structures. Journal of Applied Geophysics, 60(1), 55-67.

23. K. J. Sandmeier, (2014). REFLEXW Version 7.2.3, Program for the processing of seismic, acoustic or electromagnetic reflection, refraction and transmission data.

24. G. C. Topp, J. L. Davis, \& A. P. Annan. (1980). Electromagnetic determination of soil water content: Measurements in coaxial transmission lines. Water resources research, 16(3), 574-582. 